\title{
CONNECTED COMPONENTS IN THE INVARIABLY GENERATING GRAPH OF A FINITE GROUP
}

\author{
DANIELE GARZONI
}

(Received 21 January 2021; accepted 8 February 2021; first published online 25 March 2021)

\begin{abstract}
We prove that the invariably generating graph of a finite group can have an arbitrarily large number of connected components with at least two vertices.
\end{abstract}

2020 Mathematics subject classification: primary 20D60; secondary $20 \mathrm{E} 45$.

Keywords and phrases: connected components, invariably generating graph.

\section{Introduction}

Given a finite group $G$ and a set $X=\left\{C_{1}, \ldots, C_{t}\right\}$ of conjugacy classes of $G$, we say that $X$ invariably generates $G$ if $\left\langle x_{1}, \ldots, x_{t}\right\rangle=G$ for every $x_{1} \in C_{1}, \ldots, x_{t} \in C_{t}$. In this case, we write $\langle X\rangle_{I}=G$. This concept was introduced by Dixon [16] with motivations from computational Galois theory and has been widely studied in recent years.

In [17], the following definition was given. For a finite group $G$, the invariably generating graph $\Lambda(G)$ of $G$ is the undirected graph whose vertices are the conjugacy classes of $G$ different from $\{1\}$, and two vertices $C$ and $D$ are adjacent if $\langle C, D\rangle_{I}=G$. If $G$ is not invariably 2-generated, $\Lambda(G)$ is the empty graph. Even when $G$ is invariably 2-generated, the graph $\Lambda(G)$ can have isolated vertices (for example, when $G$ is not cyclic, the classes contained in the Frattini subgroup). Define $\Lambda^{+}(G)$ as the graph obtained by removing the isolated vertices of $\Lambda(G)$. In this paper we prove the following result.

THEOREM 1.1. For every positive integer $n$, there exists a finite group $G$ such that $\Lambda^{+}(G)$ has more than $n$ connected components.

Theorem 1.1 should be seen in comparison with the analogous graph for the case of usual generation (see Subsection 1.2).

In the proof of Theorem 1.1, we use $G=S^{\beta}$, where $S=\operatorname{PSL}_{2}(q)$ and $\beta=\beta(S)$ is the largest integer for which $S^{\beta}$ is invariably 2-generated.

The crucial observation about $S=\operatorname{PSL}_{2}(q)$ is that the graph $\Lambda^{+}(S)$ is bipartite, which follows from the fact that $S$ admits a 2-covering (Lemma 2.6). See Section 3

(C) Australian Mathematical Publishing Association Inc. 2021. This is an Open Access article, distributed under the terms of the Creative Commons Attribution licence (http://creativecommons.org/ licenses/by/4.0/), which permits unrestricted re-use, distribution, and reproduction in any medium, provided the original work is properly cited. 
for further comments in this direction, related to the clique number and the chromatic number of $\Lambda^{+}(S)$, in the case where $S$ is a nonabelian finite simple group.

In order to estimate the number of connected components of $\Lambda^{+}\left(S^{\beta}\right)$, we will need the following result.

THEOREM 1.2. Let $S=\operatorname{PSL}_{2}(q)$ and let $C_{1}, C_{2}$ be conjugacy classes of $S$ chosen uniformly at random. Then

$$
\mathbf{P}\left(\left\langle C_{1}, C_{2}\right\rangle_{I}=S\right)=1 / 2+O(1 / q) .
$$

The proof of Theorem 1.2 is straightforward, since the subgroups and conjugacy classes of $\operatorname{PSL}_{2}(q)$ are known very explicitly (and one can be much more precise about the error term).

For our application to Theorem 1.1, we could somewhat shorten the proof by imposing some restrictions on $q$ (for example, by requiring $q$ prime). However, we prefer to state and prove Theorem 1.2 in general. Indeed, it is interesting that the asymptotic behaviour of $\mathbf{P}\left(\left\langle C_{1}, C_{2}\right\rangle_{I}=S\right)$ is equal to the asymptotic behaviour of $\mathbf{P}\left(\left\langle x_{1}^{S}, x_{2}^{S}\right\rangle_{I}=S\right)$, where $x_{1}, x_{2} \in S$ are random elements (see [20, Subsection 6.1]).

1.1. Some context on $\boldsymbol{\Lambda}(\boldsymbol{G})$. Guralnick and Malle [22] and Kantor et al. [26] independently proved that every finite simple group $S$ is invariably 2-generated, so that $\Lambda(S)$ is not the empty graph. In [17], the author studied the graph $\Lambda(G)$, in the case where $G$ is an alternating or symmetric group, and proved that $\Lambda^{+}(G)$ is connected with diameter at most 6 in these cases (with the exception of $S_{6}$, which is not invariably 2-generated).

At present, it is not known whether $\Lambda^{+}(S)$ is connected if $S$ is nonabelian simple (see [17, Question 1.6]).

In [17], an analogous graph, denoted by $\Lambda_{e}(G)$, was defined. Its vertices are the nontrivial elements of $G$, and two vertices are adjacent if the corresponding classes invariably generate $G$. In [20], it was proved that if $S$ is nonabelian simple, then either $S$ belongs to three explicit families of examples, or the proportion of isolated vertices of $\Lambda_{e}(S)$ tends to zero as $|S| \rightarrow \infty$. See [20, Corollary 1.6] for a precise statement and further comments.

1.2. Comparison with usual generation. For a finite group $G$, the generating graph $\Gamma(G)$ of $G$ is the undirected graph whose vertices are the nonidentity elements of $G$, with vertices $x$ and $y$ adjacent if $\langle x, y\rangle=G$. This graph has been intensively studied in the past two decades (see Burness [10] and Lucchini and Maróti [28] for many results in this context).

Again, the graph $\Gamma(G)$ can have isolated vertices and we consider the graph $\Gamma^{+}(G)$ obtained by removing the isolated vertices of $\Gamma(G)$.

It is known that $\Gamma^{+}(G)$ is connected in several cases. For instance, Burness et al. [11] showed that if $G$ is a finite group such that every proper quotient of $G$ is cyclic, then $\Gamma(G)=\Gamma^{+}(G)$ and $\Gamma(G)$ is connected with diameter at most 2 
(the special case where $G$ is simple was proved by Guralnick and Kantor [21] and Breuer et al. [3]).

We recall other results of the same flavour, proved by Crestani and Lucchini $[12,13]$, in Section 3.

On the other hand, no example of $G$ is known for which $\Gamma^{+}(G)$ is disconnected. In fact, it is believed that there should be no such example. See Acciarri and Lucchini [1, comments after Corollary 2.7] for a more general conjecture, which would imply that $\Gamma^{+}(G)$ is connected for every 2-generated finite group $G$.

This determines a sharp difference with respect to Theorem 1.1. We note that this difference does not occur for nilpotent groups. Indeed, in a finite nilpotent group, every maximal subgroup is normal; therefore, the concepts of generation and invariable generation coincide. See Harper and Lucchini [24] for results on the generating graph of finite nilpotent groups.

\section{Proof of Theorems 1.1 and $\mathbf{1 . 2}$}

2.1. Direct powers of finite simple groups. Throughout this subsection, $S$ denotes a nonabelian finite simple group. We review some properties of invariable generation of direct powers of $S$, which reflect some interesting properties of the corresponding invariably generating graphs. The key tool is an elementary criterion due to Kantor and Lubotzky [25], which we recall here.

Denote by $\Psi_{2}(S)$ the set of all pairs $\left(C_{1}, C_{2}\right)$, where $C_{i}$ is a conjugacy class of $S$, and $\left\langle C_{1}, C_{2}\right\rangle_{I}=S$. As recalled in Subsection 1.1, it is known that $\Psi_{2}(S) \neq \varnothing$.

Now let $t$ be a positive integer and let $C$ and $D$ be conjugacy classes of $S^{t}$, with $C=C_{1} \times \cdots \times C_{t}$ and $D=D_{1} \times \cdots \times D_{t}$ (where $C_{i}$ and $D_{i}$ are conjugacy classes of $S$ ). Consider the matrix

$$
A_{C, D}=\left(\begin{array}{cccc}
C_{1} & C_{2} & \cdots & C_{t} \\
D_{1} & D_{2} & \cdots & D_{t}
\end{array}\right)
$$

LEMMA 2.1. We have $\langle C, D\rangle_{I}=S^{t}$ if and only if the following conditions are both satisfied:

(i) each column of $A_{C, D}$ belongs to $\Psi_{2}(S)$;

(ii) no two columns of $A_{C, D}$ lie in the same orbit for the diagonal action of $\operatorname{Aut}(S)$ on $\Psi_{2}(S)$.

Proof. See [25, Proposition 6] and also [14, Lemma 20].

Let $\beta=\beta(S)$ be the largest integer for which $S^{\beta}$ is invariably 2-generated. Lemma 2.1 implies that $\beta(S)$ is equal to the number of orbits for the diagonal action of $\operatorname{Aut}(S)$ on $\Psi_{2}(S)$. We note the following fact.

LEMMA 2.2. We have

$$
\frac{\left|\Psi_{2}(S)\right|}{|\operatorname{Out}(S)|} \leqslant \beta(S) \leqslant\left|\Psi_{2}(S)\right| .
$$


Proof. The second inequality is clear. The first follows from the fact that $\operatorname{Inn}(S) \cong S$ acts trivially in the relevant action, hence each orbit has size at most $|\operatorname{Out}(S)|$.

If $S$ is a nonabelian finite simple group, then $\operatorname{Out}(S)$ is 'very' small. For instance, $|\operatorname{Out}(S)|=O\left(\log _{2}|S|\right)$ (see, for example, [27, Tables 5.1.A-5.1.C] for the exact value of $|\operatorname{Out}(S)|$ in each case). We expect $|\operatorname{Out}(S)|$ to be much smaller than $\left|\Psi_{2}(S)\right|$ for every sufficiently large nonabelian finite simple group $S$. Therefore, $\left|\Psi_{2}(S)\right|$ should be, in some sense, a good approximation for $\beta(S)$.

REMARK 2.3. Let us compare Lemma 2.2 with the analogous problem for classical generation.

Let $\delta=\delta(S)$ be the largest integer for which $S^{\delta}$ is 2-generated. In contrast to the situation for $\beta(S)$, there is an exact formula for $\delta(S)$, namely, $\delta(S)=\phi_{2}(S) /|\operatorname{Aut}(S)|$, where $\phi_{2}(S)$ denotes the number of ordered pairs $(x, y) \in S^{2}$ such that $\langle x, y\rangle=S$. (This goes back to Hall [23] in the 1930s and has been widely used.) The difference is that the diagonal action of $\operatorname{Aut}(S)$ on the set of generating pairs of elements is semiregular (that is, only the identity fixes a generating pair), while this need not be the case for the action of $\operatorname{Aut}(S)$ on the set of invariable generating pairs of conjugacy classes.

Lemma 2.1 describes quite precisely the graph $\Lambda^{+}\left(S^{\beta}\right)$. Indeed, any arc in the graph is obtained as follows (and only in this way). Construct a $2 \times \beta$ matrix, in which the columns form a set of representatives for the $\operatorname{Aut}(S)$-orbits on $\Psi_{2}(S)$. Then the first row is adjacent to the second row in $\Lambda^{+}\left(S^{\beta}\right)$ (here we are identifying a conjugacy class $C_{1} \times \cdots \times C_{\beta}$ of $S^{\beta}$ with a row vector $\left.\left(C_{1}, \ldots, C_{\beta}\right)\right)$. $\operatorname{Since} \operatorname{Aut}\left(S^{\beta}\right) \cong \operatorname{Aut}(S)>\operatorname{Sym}(\beta)$ acts by automorphisms on $\Lambda^{+}\left(S^{\beta}\right)$, we also see that $\Lambda^{+}\left(S^{\beta}\right)$ is arc-transitive.

2.2. The case $S=\operatorname{PSL}_{2}(q)$. Throughout this subsection, we let $S=\operatorname{PSL}_{2}(q)$, where $q \geqslant 4$ is a power of the prime $p$, and we let $d=(2, q-1)$.

The maximal subgroups of $S$ are known from the work of Dickson [15] (see also [2, Table 8.1]). For the reader's convenience, we report here the list, up to conjugacy.

LEMMA 2.4. The maximal subgroups of $S$ are the following, up to conjugacy:

- a subgroup B of order $q(q-1) / d$ (the stabiliser of a 1-space);

- a dihedral group of order $2(q-1) / d$, for q even or $q \geqslant 13$;

- a dihedral group of order $2(q+1) / d$, for $q \neq 7,9$;

- $\operatorname{PSL}_{2}\left(q_{0}\right)$, where $q=q_{0}^{r}, q_{0} \neq 2$ and $r$ is an odd prime;

- $\operatorname{PGL}_{2}\left(q_{0}\right)$, where $q=q_{0}^{2}$ and $q_{0} \neq 2$ (two classes if $q$ is odd);

- $A_{4}$ for $q=p \equiv \pm 3,5, \pm 13(\bmod 40) ; S_{4}$ for $q=p \equiv \pm 1(\bmod 8)($ two classes $)$; $A_{5}$ for either $q=p \equiv \pm 1(\bmod 10)$, or $q=p^{2}$ and $p \equiv \pm 3(\bmod 10)($ two classes $)$.

For completeness, we report the exact conditions on $q$ under which the relevant items yield maximal subgroups of $S$, although these are largely irrelevant for our purposes. For instance, the conditions on $q$ in the last item can safely be ignored (except possibly for the purposes of Remark 2.7).

We recall other well-known facts. 
LEMMA 2.5. The following assertions hold.

(1) S contains a unique conjugacy class of involutions and, for p odd, two conjugacy classes of elements of order $p$.

(2) Let $\ell \geqslant 3$ be a divisor of $(q \pm 1) / d$. There are $\phi(\ell) / 2$ conjugacy classes of elements of order $\ell$ in $S$, where $\phi$ is Euler's totient function.

(3) The number of conjugacy classes of $S$ is $(q+4 d-3) / d$.

PROOF. We sketch a proof.

(1) Assume first that $q$ is odd, and let us deal with involutions. Let $\varepsilon=1$ if $q \equiv 1(\bmod 4)$ and $\varepsilon=-1$ otherwise. By explicit matrix computation, we find that the number of involutions of $S$ is $q(q+\varepsilon) / 2$. This coincides with the number of conjugates of a dihedral subgroup of order $q-\varepsilon$, so we deduce that all involutions of $S$ are conjugate. Next, we deal with elements of order $p$ (including the case where $q$ is even). The image in $S$ of the subgroup of $\operatorname{SL}_{2}(q)$ consisting of the upper unitriangular matrices is a Sylow $p$-subgroup $P$ of $S$. This is contained in a subgroup $B$, consisting of the image of the upper triangular matrices of $\mathrm{SL}_{2}(q)$. Let $1 \neq x \in P$. We verify that $x^{S} \cap P=x^{B}$, and we compute that conjugating $x$ by elements of $B$ can only multiply the upper-right entry by every nonzero square of $\mathbf{F}_{q}$. This proves (1).

(2) Let $\ell \geqslant 3$ be a divisor of $(q \pm 1) / d$. All cyclic subgroups of order $\ell$ are conjugate in $S$. Assume $x \in S$ has order $\ell$. Then $\mathrm{N}_{S}(\langle x\rangle)$ is dihedral of order $2(q \pm 1) / d$, from which $x^{S} \cap\langle x\rangle=\left\{x, x^{-1}\right\}$. This proves (2).

(3) An element of $S$ has either order $p$ or order dividing $(q \pm 1) / d$. Then (3) follows from (1), (2) and the formula

$$
\sum_{\ell \mid(q \pm 1) / d} \frac{\phi(\ell)}{2}=\frac{q \pm 1}{2 d} .
$$

The following lemma represents the main observation regarding $S=\operatorname{PSL}_{2}(q)$.

LEMMA 2.6. The graph $\Lambda^{+}(S)$ is bipartite.

PROOF. Let $H$ be a dihedral subgroup of $S$ of order $2(q+1) / d$, and let $K$ be a Borel subgroup of order $q(q-1) / d$. It is well known (see, for example, [6]) that $\{H, K\}$ is a 2-covering of $S$, that is,

$$
S=\bigcup_{g \in S} H^{g} \cup \bigcup_{g \in S} K^{g} .
$$

For convenience, we write $\widetilde{H}=\cup_{g \in S} H^{g}$ and $\widetilde{K}=\cup_{g \in S} K^{g}$. A conjugacy class contained in $\widetilde{H} \cap \widetilde{K}$ is isolated in $\Lambda(S)$, and a class contained in $\widetilde{H} \backslash \widetilde{K}$ can be adjacent in $\Lambda^{+}(S)$ only to a class contained in $\widetilde{K} \backslash \widetilde{H}$. This gives a partition of $\Lambda^{+}(S)$ into two parts.

We refer to Subsection 3.1 for a discussion on the topic of 2-coverings of finite simple groups. 
REMARK 2.7. Using Lemmas 2.4 and 2.5, it is not hard to show that $\Lambda^{+}(S)$ is connected with diameter at most 3. Now, let us briefly comment on the graph $\Lambda(S)$ and how it differs from $\Lambda^{+}(S)$. It is straightforward to prove the following.

(i) If $q=7$, then $\Lambda(S)$ has one isolated vertex: the class of elements of order 3 .

(ii) If $q=9$, then $\Lambda(S)$ has three isolated vertices: the two classes of elements of order 3 , and the class of involutions.

(iii) If $q \neq 9$, and $q$ is even or $q \equiv 1(\bmod 4)$ or $q \neq p$, then $\Lambda(S)$ has one isolated vertex: the class of involutions.

(iv) If $q \neq 7$ and $q=p \equiv 3(\bmod 4)$, then $\Lambda(S)$ has no isolated vertices; therefore $\Lambda(S)=\Lambda^{+}(S)$.

In particular, if $q \neq 9$ then $\Lambda(S)$ has at most one isolated vertex. This is in contrast to the case of alternating or symmetric groups $G$, where $\Lambda(G)$ can have an arbitrarily large number of isolated vertices (see [17, Theorem 1.2]).

Let $\mathscr{P}_{1}$ and $\mathscr{P}_{2}$ be the parts of $\Lambda^{+}(S)$ given in the proof of Lemma 2.6. We note that for every conjugacy class $C$ of $S$ and for every $\underset{\sim}{\sigma} \in \operatorname{Aut}(S),\left\{C, C^{\sigma}\right\}$ does not invariably generate $S$. (A way to see this is that the sets $\widetilde{H}$ and $\widetilde{K}$ from the proof of Lemma 2.6 are preserved by every automorphism of $S$.) In particular, for every $\left(C_{1}, C_{2}\right) \in \Psi_{2}(S)$, $\left(C_{1}, C_{2}\right)$ and $\left(C_{2}, C_{1}\right)$ belong to different $\operatorname{Aut}(S)$-orbits. We also note that the parts $\mathscr{P}_{1}$ and $\mathscr{P}_{2}$ are invariant under the action of $\operatorname{Aut}(S)$. We deduce the following result.

LEMMA 2.8. $\beta=\beta(S)$ is even, and for each vertex $C=C_{1} \times \cdots \times C_{\beta}$ of $\Lambda^{+}\left(S^{\beta}\right)$, there exists a subset $\Omega=\Omega(C)$ of $\{1, \ldots, \beta\}$ of size $\beta / 2$ such that $C_{i} \in \mathscr{P}_{1}$ for every $i \in \Omega$ and $C_{i} \in \mathscr{P}_{2}$ for every $i \notin \Omega$.

We can finally prove the key result.

THEOREM 2.9. The graph $\Lambda^{+}\left(S^{\beta}\right)$ has at least $\frac{1}{2} \cdot\left(\begin{array}{c}\beta \\ \beta / 2\end{array}\right)$ connected components.

Proof. For a vertex $C=C_{1} \times \cdots \times C_{\beta}$ of $\Lambda^{+}(S)$, let $\Omega(C)$ be the set from Lemma 2.8. Then, $C$ can be adjacent only to vertices $D$ such that $\Omega(D)=\{1, \ldots, \beta\} \backslash \Omega(C)$. In particular, the number of connected components of $\Lambda^{+}\left(S^{\beta}\right)$ is at least half the number of $\beta / 2$-subsets of $\{1, \ldots, \beta\}$, which proves the statement.

It is not difficult to establish that $\beta(S)$ tends to infinity as $|S| \rightarrow \infty$ (that is, $q \rightarrow \infty$ ), thereby proving Theorem 1.1. In the next subsection we will obtain a better estimate for $\beta(S)$.

2.3. Bounds. We want to estimate $\beta(S)$, where $S=\operatorname{PSL}_{2}(q)$. We will find the asymptotic behaviour of $\left|\Psi_{2}(S)\right|$ and then apply Lemma 2.2. In the following, $f=O(g)$ means that $|f| \leqslant C g$ for some constant $C$ (so $f$ might also be negative).

THEOREM 2.10. Let $S=\operatorname{PSL}_{2}(q)$ and $d=(2, q-1)$. Then

$$
\left|\Psi_{2}(S)\right|=\frac{q^{2}}{2 d^{2}}+O(q) .
$$


(For $q$ odd, the first term of the expression is not an integer, but the statement still makes sense.)

PROOF. In this proof, when we say that a conjugacy class $C$ intersects a subgroup $H$, we mean that $C \cap H \neq \varnothing$.

We need to count the pairs of conjugacy classes $\left(C_{1}, C_{2}\right)$ which invariably generate $S$. We ignore the pairs where either $C_{1}$ or $C_{2}$ comprises elements of order $p$, or order at most 2. By Lemma 2.5, the number of these pairs is $O(q)$.

By this choice, up to swapping the indices, $C_{1}$ intersects a cyclic subgroup of order $(q-1) / d$, and $C_{2}$ intersects a cyclic subgroup of order $(q+1) / d$. Given $C_{1}$ and $C_{2}$ with this property, by Lemma 2.4, we see that $C_{1}$ and $C_{2}$ invariably generate $S$ unless one of the following occurs:

(i) $C_{1}$ and $C_{2}$ intersect a subgroup isomorphic to $A_{4}, S_{4}$ or $A_{5}$, and there are at most four conjugacy classes of such subgroups;

(ii) $C_{1}$ and $C_{2}$ intersect a maximal subgroup conjugate to $\operatorname{PSL}_{2}\left(q^{1 / r}\right)$, where $r$ is an odd prime (and $q$ is an $r$ th power).

(Note that in (ii) we are excluding the subfield subgroups $\operatorname{PGL}_{2}\left(q^{1 / 2}\right)$. Indeed, any class of elements of $\mathrm{PGL}_{2}\left(q^{1 / 2}\right)$ of order prime to $q$ intersects a cyclic subgroup of $S$ of order $(q-1) / d$, and this cannot occur for $C_{2}$.) Clearly there are $O(1)$ possibilities for $\left(C_{1}, C_{2}\right)$ satisfying (i). The number of conjugacy classes of $\operatorname{PSL}_{2}\left(q^{1 / r}\right)$ is $O\left(q^{1 / r}\right)$; therefore, for fixed $r$, the number of possibilities for the pair $\left(C_{1}, C_{2}\right)$ satisfying (ii) is $O\left(q^{2 / r}\right)$. Summing over the odd prime divisors of $\log _{p} q$, we see that there are $O\left(q^{2 / 3}\right)$ possibilities in (ii), noting that there are at most $\log _{2} \log _{2} q$ possibilities for $r$.

Using Lemma 2.5, we get the following formula for $\left|\Psi_{2}(S)\right|$ (the factor 2 at the beginning comes from the fact that we may also have $C_{1}$ intersecting a cyclic subgroup of order $(q+1) / d$ and $C_{2}$ intersecting a cyclic subgroup of order $\left.(q-1) / d\right)$ :

$$
\left|\Psi_{2}(S)\right|=2 \cdot \sum_{\substack{\ell_{1}\left|(q-1) / d \\ \ell_{2}\right|(q+1) / d}} \frac{\phi\left(\ell_{1}\right)}{2} \frac{\phi\left(\ell_{2}\right)}{2}+O(q)=\frac{q^{2}-1}{2 d^{2}}+O(q)=\frac{q^{2}}{2 d^{2}}+O(q) .
$$

Here we use the formula $\sum_{\ell \mid n} \phi(\ell)=n$ and the fact that $\phi\left(\ell_{1}\right) \phi\left(\ell_{2}\right)=\phi\left(\ell_{1} \ell_{2}\right)$ for coprime $\ell_{1}$ and $\ell_{2}$.

Proof OF TheOREM 1.2. The statement follows from Lemma 2.5(3) and Theorem 2.10.

Finally, we can estimate $\beta(S)$ and obtain a lower bound for the number of connected components of $\Lambda^{+}\left(S^{\beta}\right)$, thereby proving Theorem 1.1. For aesthetic reasons, we denote $2^{a}=\exp _{2}\{a\}$, and the symbol $o(1)$ is understood with respect to the limit $q \rightarrow \infty$.

Let $N(S)$ denote the number of connected components of $\Lambda^{+}\left(S^{\beta}\right)$. 
THEOREM 2.11. If $S=\operatorname{PSL}_{2}(q)$, then

$$
\begin{gathered}
q^{2-o(1)} \leqslant \beta(S) \leqslant \frac{q^{2}}{2}+O(q) \\
N(S) \geqslant \exp _{2}\left\{q^{2-o(1)}\right\} .
\end{gathered}
$$

Proof. Equation (2.1) follows from Lemma 2.2, the fact that $|\operatorname{Out}(S)| \leqslant 2 \log _{2} q$ and Theorem 2.10. Next, Theorem 2.9, Stirling's approximation and (2.1) give

$$
N(S) \geqslant \frac{1}{2} \cdot\left(\begin{array}{c}
\beta \\
\beta / 2
\end{array}\right)=(1+o(1)) \cdot \frac{2^{\beta}}{(2 \pi \beta)^{1 / 2}} \geqslant \exp _{2}\left\{q^{2-o(1)}\right\},
$$

and the proof is complete.

\section{Further comments}

Recall that $\Gamma^{+}(G)$ is the graph obtained by removing the isolated vertices from the generating graph $\Gamma(G)$ of $G$. Crestani and Lucchini [13] showed that if $G$ is a 2-generated direct power of a nonabelian finite simple group, then $\Gamma^{+}(G)$ is connected.

In particular, Theorem 2.11 shows that the result of [13] does not hold for invariable generation. Nevertheless, a combinatorial proof along the lines of [13, Theorem 3.1] might be feasible in order to show the following: if a finite simple group $S$ is such that $\Lambda^{+}(S)$ is connected and not bipartite, then $\Lambda^{+}\left(S^{t}\right)$ is connected for every $t \leqslant \beta(S)$. At present, the connectedness of $\Lambda^{+}(S)$ for $S$ simple is essentially only known for alternating groups [17], which somewhat limits the applications of such a result.

We also remark that we are currently unable to construct examples of soluble groups $G$ for which $\Lambda^{+}(G)$ is disconnected.

QUESTION 3.1. Let $G$ be a finite soluble group which is invariably 2-generated. Is the graph $\Lambda^{+}(G)$ connected?

Crestani and Lucchini [12] showed that this is true for the graph $\Gamma^{+}(G)$ (and, in particular, Question 3.1 has a positive answer for nilpotent groups).

3.1. $\boldsymbol{\Lambda}^{+}(\boldsymbol{S})$ bipartite. For the proof of Theorem 2.11 , the only important property of $S=\operatorname{PSL}_{2}(q)$ is that the graph $\Lambda^{+}(S)$ is bipartite, which follows from the fact that $S$ admits a 2-covering (see the proof of Lemma 2.6).

The 2-coverings of the finite simple groups have been well studied (see Bubboloni [5], Bubboloni and Lucido [6], Bubboloni et al. [7, 8] and Pellegrini [29]). In particular, all finite simple groups admitting a 2-covering are known, except for some classical groups of small dimension.

We have the following clear implications:

$S$ admits a 2-covering $\Longrightarrow \Lambda^{+}(S)$ is bipartite $\Longrightarrow \Lambda^{+}(S)$ has no triangles.

(These implications are a special case of the inequalities in (3.2) below.) The reverse of the first implication in (3.1) does not necessarily hold. For instance, $A_{9}$ does not 
admit a 2-covering. (It was proved in [5] that $A_{n}$ admits a 2-covering if and only if $4 \leqslant n \leqslant 8$.) On the other hand, it is not difficult to show that $\Lambda^{+}\left(A_{9}\right)$ is bipartite. This might be one of only finitely many exceptions.

Problem 3.2. Determine the finite simple groups $S$ for which $\Lambda^{+}(S)$ is bipartite (respectively, contains no triangles). Up to finitely many cases, do the reverse implications in (3.1) hold?

These considerations can be viewed more generally as follows. For a noncyclic finite group $G$, consider the following invariants:

- $\kappa(G)$, the clique number of $\Lambda^{+}(G)$, that is, the largest order of a complete subgraph of $\Lambda^{+}(G)$;

- $\tau(G)$, the chromatic number of $\Lambda^{+}(G)$, that is, the least number of colours needed to colour the vertices of $\Lambda^{+}(G)$ in such a way that adjacent vertices have different colours;

- $\gamma(G)$, the normal covering number of $G$, that is, the least number of proper subgroups of $G$ such that each element of $G$ lies in some conjugate of one of these subgroups.

The following inequalities hold:

$$
\kappa(G) \leqslant \tau(G) \leqslant \gamma(G)
$$

(These are 'invariable' versions of inequalities studied, for instance, in [28].) The implications in (3.1) can be stated as follows for a general noncyclic finite group $G$ :

$$
\gamma(G) \leqslant 2 \Longrightarrow \tau(G) \leqslant 2 \Longrightarrow \kappa(G) \leqslant 2 .
$$

(We note that for every finite group $G, \gamma(G) \geqslant 2$ and, by [22, 26], if $S$ is nonabelian simple then $\kappa(S) \geqslant 2$.) Problem 3.2 asks whether, up to finitely many exceptions,

$$
\gamma(S)=2 \Longleftrightarrow \tau(S)=2 \Longleftrightarrow \kappa(S)=2 .
$$

The invariants $\kappa(G)$ and $\gamma(G)$ are studied in Britnell and Maróti [4], Bubboloni et al. [9] and Garonzi and Lucchini [18].

As a final remark, the fact that $\Lambda(G)$ can have no triangles is somewhat strange, in comparison with classical generation. Indeed, for every 2-generated finite group $G$ of order at least 3, the generating graph $\Gamma(G)$ contains a triangle, and indeed 'many' triangles. This follows from the fact that if $\langle x, y\rangle=G$ then $\langle x, x y\rangle=\langle x y, y\rangle=G$. The fact that this property fails for invariable generation makes it difficult to extend results from the classical to the invariable setting. See the introduction of [19] for comments in this direction.

\section{Acknowledgements}

The author thanks Andrea Lucchini for a discussion about the literature on the generating graph of a finite group, and Daniela Bubboloni for a discussion about the literature on normal coverings of finite simple groups. 


\section{References}

[1] C. Acciarri and A. Lucchini, 'Graphs encoding the generating properties of a finite group', Math. Nachr. 293(9) (2020), 1644-1674.

[2] J. N. Bray, D. F. Holt and C. M. Roney-Dougal, The Maximal Subgroups of the Low-Dimensional Finite Classical Groups, London Mathematical Society Lecture Note Series, 407 (Cambridge University Press, Cambridge, 2013).

[3] T. Breuer, R. M. Guralnick and W. M. Kantor, 'Probabilistic generation of finite simple groups. II', J. Algebra 320(2) (2008), 443-494.

[4] J. Britnell and A. Maróti, 'Normal coverings of linear groups', Algebra Number Theory 7(9) (2013), 2085-2102.

[5] D. Bubboloni, 'Coverings of the symmetric and alternating groups', Preprint, 2010, arXiv: 1009.3866.

[6] D. Bubboloni and M. S. Lucido, 'Coverings of linear groups', Comm. Algebra 30(5) (2002), 2143-2159.

[7] D. Bubboloni, M. S. Lucido and T. Weigel, 'Generic 2-coverings of finite groups of Lie type', Rend. Semin. Mat. Univ. Padova 115 (2006), 209-252.

[8] D. Bubboloni, M. S. Lucido and T. Weigel, '2-coverings of classical groups', Preprint, 2011, arXiv: 1102.0660.

[9] D. Bubboloni, C. E. Praeger and P. Spiga, 'Normal coverings and pairwise generation of finite alternating and symmetric groups', J. Algebra 390 (2013), 199-215.

[10] T. C. Burness, 'Simple groups, generation and probabilistic methods', Groups St Andrews 2017 in Birmingham, London Mathematical Society Lecture Note Series, 455 (Cambridge University Press, Cambridge, 2019), 200-229.

[11] T. C. Burness, R. M. Guralnick and S. Harper, 'The spread of a finite group', Ann. of Math. 193(2) (2021), 619-687.

[12] E. Crestani and A. Lucchini, 'The generating graph of finite soluble groups', Israel J. Math. 198(1) (2013), 63-74.

[13] E. Crestani and A. Lucchini, 'The non-isolated vertices in the generating graph of a direct powers of simple groups', J. Algebraic Combin. 37(2) (2013) 249-263.

[14] E. Detomi and A. Lucchini, 'Invariable generation with elements of coprime prime-power orders', J. Algebra 423 (2015), 683-701.

[15] L. E. Dickson, Linear Groups: With an Exposition of the Galois Field Theory (Dover Publications, New York, 1958).

[16] J. D. Dixon, 'Random sets which invariably generate the symmetric group', Discrete Math. 105(1-3) (1992), 25-39.

[17] D. Garzoni, 'The invariably generating graph of the alternating and symmetric groups', J. Group Theory 23(6) (2020), 1081-1102.

[18] M. Garonzi and A. Lucchini, 'Covers and normal covers of finite groups', J. Algebra 422 (2015), $148-165$.

[19] D. Garzoni and A. Lucchini, 'Minimal invariable generating sets', J. Pure Appl. Algebra 224(1) (2020), 218-238.

[20] D. Garzoni and E. McKemmie, 'On the probability of generating invariably a finite simple group', Preprint, 2020, arXiv:2008.03812.

[21] R. M. Guralnick and W. M. Kantor, 'Probabilistic generation of finite simple groups', J. Algebra 234(2) (2020), 743-792.

[22] R. M. Guralnick and G. Malle, 'Simple groups admit Beauville structures', J. Lond. Math. Soc. (2) 85(3) (2012), 694-721.

[23] P. Hall, 'The Eulerian functions of a group', Q. J. Math. os-7(1) (1936), 134-151.

[24] S. Harper and A. Lucchini, 'Connectivity of generating graphs of nilpotent groups', Algebraic Combin. 3 (2020), 1183-1195. 
[25] W. M. Kantor and A. Lubotzky, 'The probability of generating a finite classical group', Geom. Dedicata 36(1) (1990), 67-87.

[26] W. M. Kantor, A. Lubotzky and A. Shalev, 'Invariable generation and the Chebotarev invariant of a finite group', J. Algebra 348 (2011), 302-314.

[27] P. Kleidman and M. W. Liebeck, The Subgroup Structure of the Finite Classical Groups, London Mathematical Society Lecture Note Series, 129 (Cambridge University Press, Cambridge, 1990).

[28] A. Lucchini and A. Maróti, 'Some results and questions related to the generating graph of a finite group', Ischia Group Theory 2008 (World Scientific, Singapore, 2009), 183-208.

[29] M. A. Pellegrini, '2-coverings for exceptional and sporadic simple groups', Arch. Math. 101(3) (2013), 201-206.

DANIELE GARZONI, Dipartimento di Matematica 'Tullio Levi-Civita', Università degli Studi di Padova, Padova, Italy

e-mail: daniele.garzoni@phd.unipd.it 